\title{
Laparoscopic Roux-en-Y Gastric Bypass After Failed Vertical Banded Gastroplasty: a Multicenter Experience with 203 Patients
}

\author{
M. Suter • S. Ralea $\cdot$ P. Millo • J. L. Allé
}

Published online: 15 June 2012

(C) Springer Science+Business Media, LLC 2012

\begin{abstract}
Background Vertical banded gastroplasty (VBG) has long been the main restrictive procedure for morbid obesity but has many long-term complications for which conversion to Roux-en-Y gastric bypass (RYGBP) is often considered the best option.

Methods This series regroups patients operated on by three different surgeons in four different centers. All data were collected prospectively, then pooled and analyzed retrospectively.

Results Out of 2,522 RYGBP performed between 1998 and 2010, 538 were reoperations, including 203 laparoscopic RYGBP after VBG. There were 175 women and 28 men. The mean BMI before VBG was $43.2 \pm 6.3$, and the mean BMI before reoperation was $37.4 \pm 8.3$. Most patients had more than one indication for reoperation and/or had regained significant weight. There was no conversion to open surgery. A total of 24 patients $(11.8 \%)$ developed complications, including nine (4.5\%) who required reoperation and one death. With a follow-up of $88.9 \%$ after
\end{abstract}

\footnotetext{
M. Suter $(\bowtie)$

Department of Surgery, Hôpital du Chablais,

1860 Aigle-Monthey, Switzerland

e-mail: michelsuter@netplus.ch

M. Suter

Department of Visceral Surgery, CHUV,

Lausanne, Switzerland

P. Millo

Department of Surgery, Ospedale Umberto Parini,

Aosta, Italy

S. Ralea · J. L. Allé

Department of Surgery, CHU Tivoli,

La Louvière, Belgium

8 years, the mean BMI after 1, 3, 5, 7, and 9 years was $29.1,28.8,28.7,29.9$, and 28.8, respectively.

Conclusions On the basis of this experience, the largest with laparoscopic reoperative RYGBP after failed VBG, we conclude that this procedure can safely be performed in experienced hands, with weight loss results similar to those observed after primary RYGBP. In patients with too difficult an anatomy below the cardia, dividing the esophagus just above the esophago-gastric junction and performing an esophagojejunostomy may be a safe alternative to converting to a Scopinaro-type BPD, obviating the additional longterm risks associated with malabsorption.

Keywords Gastric bypass · Vertical banded gastroplasty . Complications $\cdot$ Reoperations $\cdot$ Laparoscopy $\cdot$ Weight loss

\section{Introduction}

The prevalence of obesity has been growing constantly over the past 20 years, resulting in an increasing number of patients undergoing bariatric surgery [1]. In Europe, restrictive procedures such as vertical banded gastroplasty (VBG) and later gastric banding (GB) have been the procedures of choice until the beginning of this century, when Roux-en-Y gastric bypass (RYGBP) became progressively more popular and more recently when sleeve gastrectomy was introduced.

Restrictive bariatric surgery is associated with a relatively high long-term failure rate and a high incidence of long-term complications. After VBG, restriction is sometimes excessive because of too narrow a band and/or the development of a stricture. This leads many patients to slowly change their dietary habits to a soft semi-liquid energy-rich diet, which can cause important weight regain $[2,3]$. Others 
tolerate severe restriction less and less, depend on anti-reflux medications, or even continue to lose weight until they become underweight. Other late complications are pouch dilation or staple line disruption. Studies on long-term results after VBG show that up to more than $50 \%$ of the patients eventually need redo surgery [3-8]. While restoration of the initial procedure can sometimes be done safely, it has been shown that conversion to RYGBP provides better results, better weight loss or maintenance, and fewer further complications leading to fewer further reoperations [7, 9-12].

A history of former VBG, especially when done openly, is considered by many as a relative contra-indication to a laparoscopic approach for conversion to RYGBP. Indeed the experience reported in the literature with the latter has been very limited, with only 11 papers, nine of them reporting on less than 20 cases [12-22]. The two largest series to date report on 51 and 105 cases, respectively, showing that laparoscopy can be used safely in this setting but failing to provide long-term results $[23,24]$. The aim of this paper is to present our common experience with a large group of more than 200 patients converted from VBG to RYGBP by laparoscopy to provide not only short- but also long-term results and to pinpoint the technical aspects and risks of the procedure.

\section{Patients and Methods}

All patients who underwent reoperative laparoscopic RYGBP after VBG by three different surgeons in one of our four centers (Department of Surgery, Regional Hospital Umberto Parini, Aosta, Italy-Department of Surgery, University Hospital La Louvière, Brussels, Belgium-Department of Visceral Surgery, University Hospital CHUV, Lausanne, Switzerland-Department of Surgery, Regional Hospital Chablais, Aigle-Monthey, Switzerland) were included in this study. Data were collected prospectively in a database in each center, transferred into a common database in Switzerland, and analyzed retrospectively.

Indication for reoperation was based on a complete reevaluation by a multidisciplinary team including a bariatric surgeon, an endocrinologist, a dietician, and in most cases a psychologist. Factors taken into account were the history of obesity before and after the initial procedure, symptoms related to complications of VBG, weight regain, and obesityrelated comorbidities. Preoperative tests included upper gastrointestinal endoscopy and an upper gastrointestinal contrast study to delineate the anatomy (size of the gastric pouch, location of the esophago-gastric junction and of the vertical staple line, hiatus hernia) or complications of the primary procedure (dehiscence of the staple line, esophagitis, stricture). Indication for reoperation was confirmed only after several visits and dietary counseling. A psychological evaluation was often undertaken, especially in patients in whom the indication for reoperation was mainly weight regain thought to be associated with eating disorders.

Data were obtained on initial weight before and maximum weight loss obtained after VBG, weight and height at reoperation, and weight at follow-up visits up to the tenth postoperative year. Previous reoperations, symptoms, and complications related to VBG were also recorded.

All procedures were performed laparoscopically. Antibiotic and thrombo-embolic prophylaxis was used routinely. A nasogastric tube was placed and the stomach was emptied before initiation of the procedure. Access to the abdominal cavity was obtained after puncture with a Verres needle or using an open approach, according to the surgeon's preference. The Verres needle was usually placed in the left upper quadrant, but in individual cases, depending on the location of previous scars, alternative sites were chosen. After placement of the initial trocar and the optic, a second trocar was placed, and lysis of adhesions between the abdominal wall and intra-abdominal content was initiated with scissors or ultrasonic shears. Once the abdominal wall was completely free of adhesions, three or four additional trocars were placed for liver retraction and operating instruments. Further adhosiolysis was then performed between the left lobe of the liver, the lesser omentum, and the anterior wall of the stomach. The right and left crus of the diaphragm were identified and the angle of His was dissected. The banding mechanism of the VBG was identified and removed, provided that it consisted of a silastic ring or an adjustable band (some patients operated on in Lausanne had an adjustable band [8]). No effort was made at removing Marlex meshes. The vertical staple line was identified. Access to the lesser sac was obtained in most cases by dissecting between the lesser omentum and the lesser curvature above the band, but in patients in whom the pouch was relatively short, dissection was initiated below the band and pursued proximally after identifying the lesser curve and the lesser sac until the upper limit of the latter was identified. Adhesions between the posterior wall of the stomach and the lesser sac were freed with caution in order to avoid any injury to the pancreas. Once preparation of the proximal stomach was deemed sufficient, formation of the new gastric pouch was initiated by transecting the stomach horizontally with a linear stapler, usually of $45 \mathrm{~mm}$ in length and with a 3.5$\mathrm{mm}$ cartridge. Care was taken not to go too close from the previous vertical staple line in order to allow drainage of the secretions from the former gastric pouch into the stomach remnant. After completing posterior dissection between the stomach and the left crus of the diaphragm, transection of the stomach was pursued vertically with a linear stapler using either 3.5- or 4.8-mm cartridges depending on the thickness of the tissue. In 16 patients, some staple line reinforcement material was used at this level. Care was 
again taken to always remain at a minimal distance from the former vertical staple line in order to avoid constructing a blind stomach pouch (Fig. 1). For the same reason, if the new vertical staple line came too close to the old one, or any doubt existed about proper drainage, the upper portion of the remnant fundus was then resected. The technique described earlier was used in the majority of patients. In some 65 of the early patients, however, and in order to avoid the fibrous tissue in the upper portion of the stomach, no attempt at forming a gastric pouch was made, and transection was performed across the esophagus immediately above the esophago-gastric junction.

A 100-150-cm Roux limb was then created after dividing the proximal jejunum between 30 and $50 \mathrm{~cm}$ distally from the angle of Treitz. Jejunojejunostomy was routinely performed using a linear stapler. The Roux limb was brought in contact with the gastric pouch in an antecolic or retrocolic way, and a gastrojejunostomy was performed, either using a circular stapler or by hand suturing, both according to the surgeon's preference. In the 65 patients with esophageal transection, a circular stapled esophagojejunostomy was constructed. A methylene blue test to check for leaks at the gastrojejunostomy was performed in the majority of patients. Except in a few early patients, all mesenteric defects were closed using a non-absorbable material, typically with running sutures. In some patients, a Jackson-Pratt drain was left in the vicinity of the gastrojejunostomy before completion of the procedure.

A water-soluble contrast study was performed during the first postoperative days. If it proved negative for any leak or stenosis, the patients were then slowly advanced on a semiliquid diet and were discharged as soon as the latter was well tolerated. Low molecular weight heparin was pursued for $2-$ 4 weeks after surgery, and proton pump inhibitors were prescribed for at least 1 month. After 1 month, solid food was progressively allowed according to the patient's tolerance, and the patients were instructed to eat slowly, to take small bites, and to chew well.

Follow-up included a first postoperative visit after 1 month. Further visits were scheduled at least bi-annually

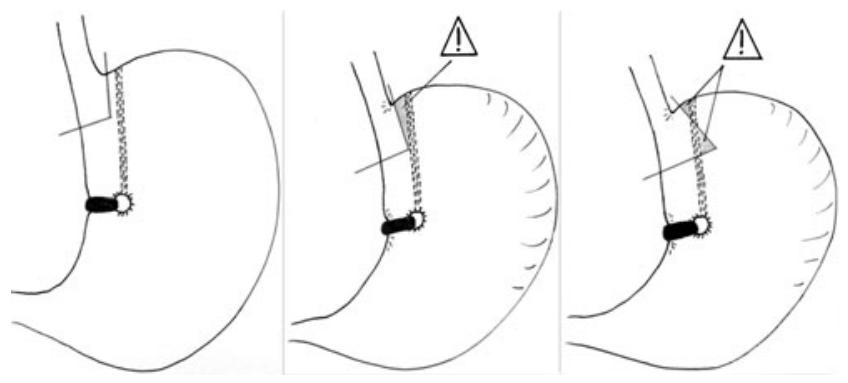

Fig. 1 Ideal placement of staple lines and places at risk from which a minimal distance must be preserved in order to avoid constructing a blind pouch until the second postoperative year, then at least annually. Patients who failed to attend scheduled visits were contacted by mail or phone and urged to do so. Follow-up evaluation included data regarding weight loss, evolution of comorbidities, and blood tests to check for possible vitamin or mineral deficiencies.

\section{Results}

A total of 2,522 RYGBP were performed in our four institutions between 1998 and December 2010. Out of these, 538 $(21.3 \%)$ were reoperations, including 203 laparoscopic reoperative RYGBP after previous VBG. There were 28 men and 175 women, with a mean age of 44.7 years $(22-68)$ at reoperation.

The mean weight (initial weight) before the first bariatric procedure (VBG) was $115.5 \pm 20.9 \mathrm{~kg}(\mathrm{SD})$, which corresponded to a mean initial BMI of $43.2 \pm 6.3 \mathrm{~kg} / \mathrm{m}^{2}$ (SD). At reoperation, the mean weight was $99.8 \pm 23.8 \mathrm{~kg}(\mathrm{SD})$, and the mean BMI was $37.4 \pm 8.27 \mathrm{~kg} / \mathrm{m}^{2}$ (SD). The main indications for reoperation are summarized in Table 1. Many patients, however, had more than one reason to be reoperated on (Table 2). Thirty-one patients had already undergone some revision of their VBG. The interval between VBG and conversion varied from one to more than 18 years, with a mean of 110 months.

All procedures were completed by laparoscopy, without conversion. The mean operative duration was $209 \mathrm{~min}$ (115-360 min). A total of 24 patients $(11.8 \%)$ developed at least one complication (Table 3), of whom nine required reoperation. There was a significant difference in the overall complication rates between the centers: 8.3 vs 27.6 vs $13.8 \%$, $p=0.01$ chi-square test), but not if only major complications are considered ( 3.4 vs 10.3 vs $3.4 \%, p=0.24$ ). Of interest is that the highest rates were noted in the center with the least overall experience in laparoscopic bariatric surgery. There was no difference in the rate of postoperative complications between patients who already had undergone revisional surgery before gastric bypass and those who had not. One patient $(0.5 \%)$ died from septic complications related to incarceration, on the third postoperative day, of an incisional hernia which had been left

Table 1 Primary reason for reoperation

\begin{tabular}{lll}
\hline Indication & Number & Percent, \% \\
\hline Weight regain & 124 & 61.1 \\
Severe late food intolerance & 46 & 22.7 \\
Gastro-esophageal reflux & 19 & 8.6 \\
Insufficient weight loss & 11 & 5.5 \\
Band slippage (Mc Lean VBG) & 2 & 1.0 \\
Band erosion & 1 & 0.5 \\
\hline
\end{tabular}


Table 2 All complications leading to reoperation after VBG

\begin{tabular}{lll}
\hline Type of complication & Number & Percent, \% \\
\hline Weight regain & 128 & 63.1 \\
Gastro-eosphageal reflux & 96 & 46.8 \\
Food intolerance & 68 & 33.5 \\
Staple line disruption & 58 & 28.6 \\
Pouch dilatation & 35 & 17.2 \\
Stricture & 22 & 10.8 \\
Insufficient weight loss & 11 & 5.5 \\
Band erosion & 4 & 2 \\
Band slippage (after Mc Lean) & 2 & 1 \\
Volvulus of the pouch & 2 & 1 \\
Esophageal dilatation & 1 & 0.5 \\
Recurrence of comorbidity (diabetes) & 1 & 0.5 \\
Large para-esophageal hernia & 1 & 0.5 \\
\hline
\end{tabular}

unrepaired at the time of surgery. Another patient, who developed leaks both at the gastrojejunostomy and the jejunojejunostomy, required several reoperations but was eventually discharged after 60 days.

At the time of this writing, duration of follow-up was at least 3 years in 153 patients, at least 5 years in 94, and at least 8 years in 27. At the same time intervals, 79.1, 90.4 and $88.9 \%$ of the eligible patients, respectively, were available for follow-up. Results in terms of weight loss are depicted in Figs. 2, 3, 4, and 5. Figure 2 shows the evolution of excess BMI loss (EBMIL) in relation to the initial BMI, and Fig. 3 shows the evolution of the mean BMI. Figure 4 shows the distribution of BMI over time. Patients who underwent reconstruction with esophagojejunostomy maintained a similar or even better weight loss throughout the study period when compared with those in whom a small gastric pouch was constructed and who had a gastrojejunostomy as in standard RYGBP (Fig. 5). Late complications developed in

Table 3 Early complications

\begin{tabular}{lll}
\hline Type of complications & Number & Percent, \% \\
\hline Leak at the gastrojejunostomy & 6 & 2.9 \\
Leak at the jejunojejunostomy & 2 & 1 \\
Intestinal obstruction & 4 & 1.9 \\
Intra-abdominal abscess & 1 & 0.5 \\
Intraluminal hemorrhage & 2 & 1 \\
Urinary tract infection & 1 & 0.5 \\
Bronchopneumonia & 3 & 1.5 \\
Trocar site infection & 1 & 0.5 \\
Respiratory failure & 1 & 0.5 \\
Intraoperative diaphragmatic tear & 1 & 0.5 \\
Others & 2 & 1 \\
Total patients with complication(s) & 24 & 11.8 \\
\hline
\end{tabular}

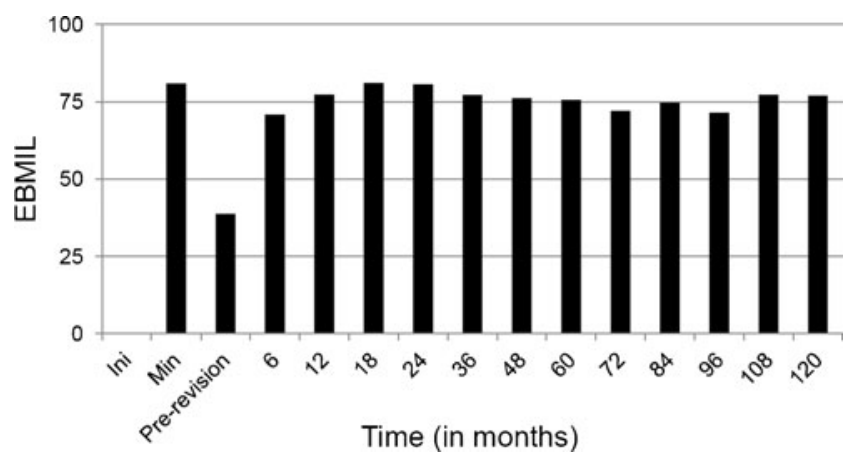

Fig. 2 Evolution of the percentage of excess BMI loss (EBMIL) in relation to the initial (pre-VBG) BMI. Ini pre-VBG, Min minimum EBMIL obtained at any time after VBG but before revision, Prerevision EBMIL at revision

$23(11.6 \%)$ patients and are detailed in Table 4. Fourteen $(7.1 \%)$ of these patients required surgery for correction.

\section{Discussion}

VBG was introduced in the late 1970 s as an alternative to gastric bypass [25] in an attempt to provide similar weight loss with less morbidity. Despite its wide initial adoption, and satisfactory early results, VBG caused a high proportion of long-term complications, both anatomic (staple line disruption, stricture of the stoma, pouch dilatation) and functional (severe food intolerance, gastresophageal reflux disease). Also, many patients tended progressively to modify their eating habits and switch to a liquid or semi-liquid energy-rich diet. Along with these complications or dietary changes, several patients progressively regained weight. During the late 1980s and early 1990s, several randomized studies have shown the superiority of gastric bypass over VBG [2, 26-30] not only in terms of weight loss but also with respect to long-term morbidity. VBG, however, has remained as one of the most

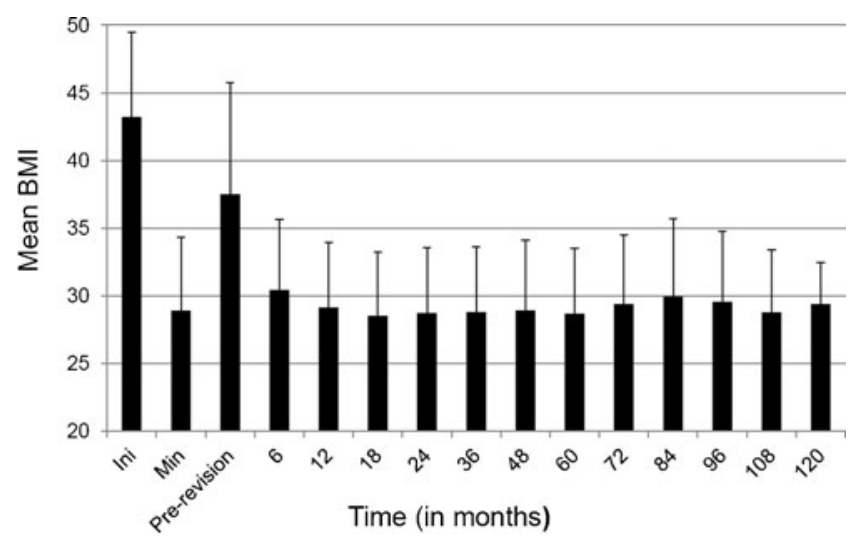

Fig. 3 Evolution of the BMI $( \pm \mathrm{SD})$ over time. Ini pre-VBG, Min minimum BMI obtained at any time after VBG but before revision, Pre-revision BMI at revision 


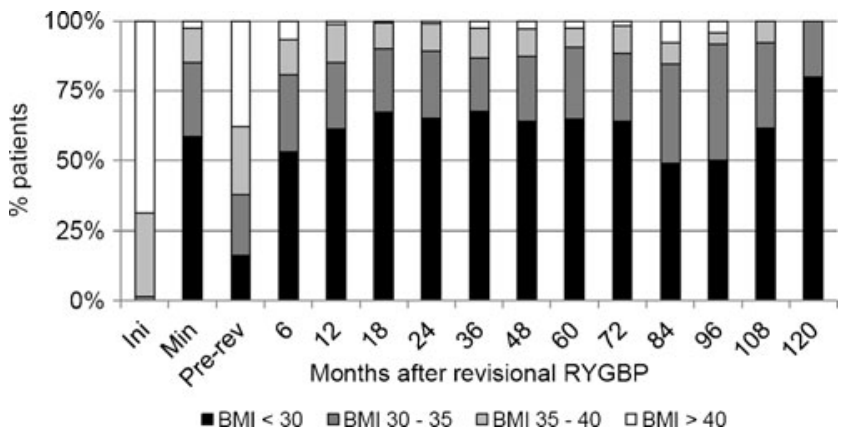

Fig. 4 Evolution of the distribution of BMI over time

commonly performed bariatric operations in several European countries, particularly in Switzerland and Belgium, and laparoscopic techniques have been developed in the 1990s $[31,32]$ until it was largely replaced as a purely restrictive procedure by laparoscopic gastric banding.

Several complications of VBG eventually require surgical correction because of weight regain or severely impaired quality of life. Revisional bariatric surgery is usually considered as risky and has been associated with relatively high complication and mortality rates compared with primary bariatric surgery. Morbidity rates in the range of 20-50\% have regularly been reported after open revision [9-12, 33-35]. After VBG, revision can include repair of the anatomical defect (i.e., re-stapling for staple line dehiscence), conversion to another procedure, or even simple reversal, although the latter is almost inevitably associated with progressive weight regain. Whereas correction of the anatomical defect is usually possible when complications arise after VBG, several authors have shown conversion to RYGBP as a more effective re-do procedure in these cases because it leads to better weight loss and maintenance and is associated with fewer further long-term complications, hence the reduced need for repeated re-do surgery [9-12].

Revisional bariatric surgery is technically challenging. Numerous difficulties are related to the altered anatomy and adhesions, and identifying previous staple lines is not

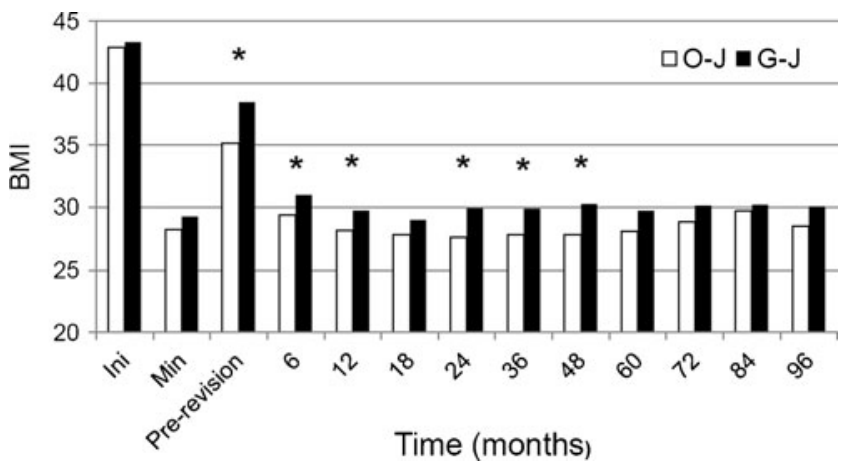

Fig. 5 Comparison of the BMI after revisional RYGBP between patients with esophagojejunostomy $(O-J)$ and those with a gastric pouch and gastrojejunostomy $(G-J) .{ }^{*} p<0.05$
Table 4 Long-term complications

\begin{tabular}{lll}
\hline Type of complication & Number & Percent, \% \\
\hline Stricture at the gastrojejunostomy & 3 & 1.5 \\
Marginal ulcer & 7 & 3.5 \\
Internal hernia & 5 & 2.5 \\
Bowel occlusion (adhesions) & 2 & 1.0 \\
Trocar site hernia & 1 & 0.5 \\
Incisional hernia (after laparotomy) & 1 & 0.5 \\
Intussusception & 1 & 0.5 \\
Strangulated umbilical hernia & 1 & 0.5 \\
Gastro-gastric fistula & 1 & 0.5 \\
Gastro-cutaneous fistula & 1 & 0.5 \\
Symptomatic gallstones & 1 & 0.5 \\
Recurrent abdominal pain & 2 & 1.0 \\
\hline
\end{tabular}

always easy, especially after undivided VBG. The presence of fibrotic tissues makes staple lines fragile and more prone for leakage. It has been shown that multiple prior procedures increase the risks to develop postoperative complications and especially major morbidity [36]. In the largest series reported so far, including 218 patients all reoperated by laparotomy except for two, the mean operative time was almost $5 \mathrm{~h}$, and serious postoperative complications developed in $26 \%$ of the patients, with a $0.9-\%$ mortality and a 9day mean postoperative stay [12]. With the hope that the reduced operative trauma, together with the magnification provided by the laparoscopic approach, would contribute in decreasing the overall operative risks and shorten the hospital stay, several authors have attempted to perform these difficult reoperations using a laparoscopic approach. The first successful experience, reported 10 years ago, included seven reoperative RYGBP, six after previous open VBG [13]. Isolated case reports or small series reported within the next few years, typically including patients with various primary procedures, confirmed the feasibility and safety of the laparoscopic approach for reoperative RYGBP, with a reduced overall morbidity (from 0 to $22 \%$ ) and a much lower major complication rate (0-12.5\%) compared with the figures reported after open redo surgery [14-16, 18, 37, 38]. In series with a high conversion rate, however, overall morbidity remained high [19].

Revisional RYGBP after VBG is considered to be more challenging than after $\mathrm{GB}$, one obvious reason for this being the fact that the majority of VBG have been performed through laparotomy, whereas most GB were done by laparoscopy. Additionally, the dissection required for VBG is more extensive, and the latter includes permanent staple lines. Most published series on laparoscopic revisional RYGBP include mostly patients with prior GB and only a small number of patients after VBG, with 80 cases distributed among nine series [12-22, 39]. Cadière et al. compared the results of 362 
primary RYGBP with 108 revisions, including 51 patients who originally had a VBG [23]. Operative time was longer (194 versus $109 \min , p<0.001$ ) and overall morbidity was higher $(22.2$ versus $10.2 \%, p<0.001)$ in reoperated patients, especially with respect to leaks at the gastrojejunostomy (12 versus $4.4 \%, p=0.004$ ), a difference the authors attribute to fibrosis and ischemia related to more extensive dissection. The largest experience with laparoscopic revisional RYGBP after VBG has been reported last year by Gagné et al. [24] with a series including 105 patients. Their median operative time (175 min) was slightly shorter than ours. There was no mortality, although early complications developed in 20 patients (19\%), of whom ten $(9.5 \%)$ required reoperation. Our $11.8 \%$ early complication rate compares very well with that reported in both of these series. It is also lower than that seen after open revisional RYGBP. In fact, it is not much higher than the complication rate seen after primary gastric bypass in several recent reports [40-43]. The differences we observed in our complication rates between centers, with the highest rate in the center with the least bariatric experience (although the surgeon had been a leading expert in advanced laparoscopic surgery for several years), underline the fact that these difficult procedures should only be performed by very experienced not only laparoscopic but also bariatric surgeons. As in the two aforementioned reports, our commonest complication was leak at the gastrojejunostomy, the incidence of which (2.9\%) is twice as high as that after primary RYGBP. This is probably related to tissue scarring and fibrosis in the area of the proximal stomach and ischemia related to extensive dissection. Although we try to always avoid stapling in obviously abnormal tissue and/or across previous staple lines, we usually use higher staple height (4.8 versus $3.5 \mathrm{~mm}$ ) for division of the proximal stomach in redo patients to accommodate for the greater tissue thickness. We also fire the staplers very slowly to allow tissue fluid to move away from the staple line, which improves closure of the staples. We have recently introduced staple line reinforcement to further reduce our leak rate but cannot yet conclude whether this precaution is of any benefit.

The only patient who died in our experience did so from the septic consequences of small bowel obstruction and necrosis due to an unrecognized incarcerated incisional hernia. Another patient who developed the same complication was diagnosed and reoperated rapidly, with good outcome. In asymptomatic patients with incisional hernia after previous surgery, omental fat usually fills the defect(s). Once the adhesions have been taken down, and if the defect is not repaired, there is a great risk of repeated herniation. The altered anatomy after gastric bypass increases not only the risk of small bowel herniation but also the difficulties in diagnosing small bowel obstruction [44]. In order to avoid this risk, some authors advocate incisional hernia repair at the end of the procedure, even if a mesh has to be used [44, 45]. If hernia sites are not repaired before completing revisional gastric bypass, a great index of suspicion must exist if anything goes wrong, and the possibility of incarceration must be kept in mind. We recently had to reoperate on a redo patient (not included in this series) on the first postoperative day because of an incarcerated small bowel hernia. The hernia was reduced and laparoscopic repair with a mesh was successfully performed.

In order to avoid acute dilatation of the gastric remnant in the setting of postoperative obstruction, some authors advocate the routine placement of a gastrostomy tube, which can also be used for enteral feeding if required [24]. We do not believe this to be necessary in the majority of patients since postoperative obstruction is unusual. It would unnecessarily expose many patients to the potential complications of gastrostomy. We strongly suggest placing one, however, in patients who require early revision because of postoperative occlusion.

In Cadière's experience, long-term complications were also more frequent after reoperative RYGBP, with a higher anastomotic stricture rate (18.5 versus $5.5 \%, p<0.001)$, especially after GB (26.1 versus $7 \%$ after VBG, $p=0.01)$. Long-term results in terms of weight loss, however, were similar to those obtained after primary RYGBP [23]. Stricture was again the commonest late complication in Gagné's experience [24]. In our series, only three patients developed anastomotic stricture, all treated by endoscopic dilation. We have no explanation for this low rate, which may be related to the surgical technique used for gastrojejunostomy (mechanical circular stapled anastomosis in most patients).

Gastric mucocele can develop as a complication of revision after VBG, and it has been reported after both conversion to RYGBP or to duodenal switch [22, 46, 47]. It results from stapling too close to the previous staple line or even across it, thereby forming a closed pouch with no communication with the rest of the stomach (pouch or remnant). When dividing the proximal stomach, great care must be taken to identify the former vertical staple line and to remain on its right side, leaving at least a few millimeters between the edge of the pouch and the former staple line so that any small gastric portion left on the right of the former staple line is drained into the former pouch and through the outlet of the former gastroplasty (Fig. 1). In case of doubt, one must not hesitate to resect the upper portion of the remnant, including the upper portion of the former staple line.

We seem to be the first group to report on long-term results after revisional gastric bypass. Long-term weight loss is comparable to that seen after primary gastric bypass, with a mean BMI remaining around 30 up to 10 years after revision. The number of patients with very long follow-up, however, is still limited so that definitive conclusions about long-term weight loss should be interpreted with caution.

Interestingly, patients operated early on with an esophagojejunostomy maintained a lower mean BMI than their counterparts with a pouch and a standard gastrojejunostomy 
throughout the study period, although the difference lost statistical significance beyond 48 months. In patients with a difficult anatomy at the upper portion of the stomach, with important scarring and fibrosis, conversion to a Scopinarotype biliopancreatic diversion, which obviates dissection in this area, is sometimes regarded as a safer option than conversion to a standard gastric bypass. From our experience, however, we conclude that dividing the esophagus just above the cardia and performing an esophagojejunostomy may be a safe alternative to conversion to BPD. The overall complication rate is similar ( 9.2 versus $13 \%, p=0.43)$, the incidence of leaks is the same, and it does not jeopardize the results of RYGBP in terms of weight loss.

\section{Conclusions}

On the basis of this experience, the largest so far with laparoscopic reoperative RYGBP after failed VBG, we conclude that this procedure can safely be performed in experienced hands, with an acceptable morbidity and mortality. Results in terms of weight loss are similar to those observed after primary RYGBP [31]. In patients with too difficult an anatomy just below the cardia, dividing the esophagus just above the esophago-gastric junction and performing an esophagojejunostomy may be a safe alternative to converting to a Scopinaro-type BPD, obviating the additional longterm risks associated with malabsorption.

Disclosure None of the authors has any conflict of interest in relation with this manuscript.

\section{References}

1. Buchwald H, Oien DM. Metabolic/bariatric surgery worldwide 2008. Obes Surg. 2009;19:1605-11.

2. Brolin RL, Robertson LB, Kenler HA, et al. Weight loss and dietary intake after vertical banded gastroplasty and Roux-en-Y gastric bypass. Ann Surg. 1994;220:782-90.

3. Shai I, Henkin Y, Weitzman S, Levi I. Long-term dietary changes after vertical banded gastroplasty: is the trade-off favorable? Obes Surg. 2002;12:805-11.

4. Schouten R, Wiryasaputra DC, Van Dielen FM, et al. Long-term results of bariatric restrictive procedures: a prospective study. Obes Surg. 2010;20:1617-26.

5. Marsk R, Jonas E, Gartzios H, et al. High revision rates after laparoscopic vertical banded gastroplasty. Surg Obes Relat Dis. 2009;5:94-8.

6. Miller K, Pump A, Hell E. Vertical banded gastroplasty versus adjustable gastric banding: prospective long-term follow-up study. Surg Obes Relat Dis. 2007;3:84-90.

7. Del Amo DA, Diez MM, Guedea ME, et al. Vertical banded gastroplasty: is it a durable operation for morbid obesity? Obes Surg. 2004;14:536-8.
8. Suter M, Jayet C, Jayet A. Vertical banded gastroplasty: long-term results comparing three different techniques. Obes Surg. 2000;10:41-6.

9. Behrns KE, Smith D, Kelly KA, et al. Reoperative bariatric surgery. Lessons learned to improve patient selection and results. Ann Surg. 1993;218:646-553.

10. Benotti PN, Forse RA. Safety and long-term efficacy of revisional surgery in severe obesity. Am J Surg. 1996;172:232-5.

11. Van Gemert WG, Van Wersch MM, Greve JWM, et al. Revisional surgery after failed vertical banded gastroplasty: restoration of vertical banded gastroplasty or conversion to gastric bypass. Obes Surg. 1998;8:21-8.

12. Nesset EM, Kendrick ML, Houghton SG, et al. A two-decade spectrum of revisional surgery at a tertiary referral center. Surg Obes Realt Dis. 2007;3:25-30.

13. De Csepel J, Nahouraii R, Gagner M. Laparoscopic gastric bypass as a reoperative bariatric surgery for failed open restrictive procedures. Surg Endosc. 2001;15:393-7.

14. Cohen R, Pinheiro JS, Correa JL, et al. Laparoscopic revisional bariatric surgery. Myths and facts. Surg Endosc. 2005;19:822-5.

15. Calmes JM, Giusti V, Suter M. Reoperative laparoscopic Roux-enY gastric bypass: an experience with 49 cases. Obes Surg. 2005;15:316-22.

16. Gagner M, Gentileschi P, de Csepel J, et al. Laparoscopic reoperative bariatric surgery. Experience from 27 consecutive patients. Obes Surg. 2002;12:254-60.

17. Khaitan L, Van Sickle K, Gonzalez R, et al. Laparoscopic revision of bariatric procedures: is it feasible? Am Surg. 2005;71:6-12.

18. Bloomberg RD, Urbach DR. Laparoscopic Roux-en-Y gastric bypass for severe gastroesophageal reflux after vertical banded gastroplasty. Obes Surg. 2002;12:408-11.

19. Van Dessel E, Hubens G, Ruppert M, et al. Roux-en-Y gastric bypass as a re-do procedure for failed restrictive gastric surgery. Surg Endosc. 2008;22:1014-8.

20. Moreno-Portillo M, Pereira-Graterol F, Magos FJ, et al. Conversion to laparoscopic Roux-en-Y gastric bypass after vertical banded gastroplasty. A case report. Cir Cir. 2004;72:131-4.

21. Ianelli A, Amato D, Addeo P, et al. Laparoscopic conversion of vertical banded gastroplasty (Mason MacLean) into Roux-en-Y gastric bypass. Obes Surg. 2008;18:43-6.

22. Mognol P, Chosidow D, Marmuse JP. Roux-en-Y gastric bypass after failed vertical banded gastroplasty. Obes Surg. 2007;17:1431-4.

23. Cadière GB, Himpens J, Bazi M, et al. Are laparoscopic gastric bypass after gastroplasty and primary laparoscopic gastric bypass similar in terms of results? Obes Surg. 2011;21:692-8.

24. Gagné DJ, Dovec E, Urbandt JE. Laparoscopic revision of vertical banded gastroplasty to Roux-en-Y gastric bypass. Surg Obes Relat Dis. 2011;7:493-9.

25. Mason EE. Vertical banded gastroplasty for obesity. Arch Surg. 1982;117:701-6.

26. Sugerman HJ, Londrey GL, Kellum JM, et al. Weight loss with vertical banded gastroplasty and Roux-en-Y gastric bypass for morbid obesity with selective versus random assignment. Am J Surg. 1989;157:93-102.

27. Sugerman HJ, Starkey JV, Birkenhauer R. A randomized prospective trial of gastric bypass versus vertical banded gastroplasty for morbid obesity and their effects on sweets versus non-sweets eaters. Ann Surg. 1987;205:613-24.

28. Fobi MA. Vertical banded gastroplasty vs gastric bypass: 10 years follow-up. Obes Surg. 1993;3:161-4.

29. Capella JF, Capella RF. The weight reduction operation of choice: vertical banded gastroplasty or gastric bypass? Am J Surg. 1996;171:74-9.

30. Howard L, Malone M, Michalek A, et al. Gastric bypass and vertical banded gastroplasty - a prospective randomized comparison and 5-year follow-up. Obes Surg. 1995;5:55-60. 
31. Lönroth H, Dalenbäck J, Haglind E, et al. Vertical banded gastroplasty by laparoscopic technique in the treatment of morbid obesity. Surg Laparosc Endosc. 1996;6:102-7.

32. Catona A, Gossenberg M, Mussini G, et al. Videolaparoscopic vertical banded gastroplasty. Obes Surg. 1995;5:323-6.

33. Cordera F, Mai JL, Thompson GB, et al. Unsatisfactory weight loss after vertical banded gastroplasty: is conversion to Roux-en-Y gastric bypass successful? Surgery. 2004;136:737-7.

34. Balsiger BM, Murr MM, Mai J, et al. Gastroesophageal reflux after intact vertical banded gastroplasty: correction by conversion to Roux-en-Y gastric bypass. J Gastrointest Surg. 2000;4:276-81.

35. Cariani S, Nottola D, Grani S, et al. Complications after gastroplasty and gastric bypass as a primary operation and as a reoperation. Obes Surg. 2001;11:487-90.

36. Roller JE, Provost DA. Revision of failed gastric restrictive operations to Roux-en-Y gastric bypass: impact of multiple prior bariatric operations on outcome. Obes Surg. 2006;16:865-9.

37. Mognol P, Chosidow D, Marmuse JP. Laparoscopic conversion of laparoscopic gastric banding to Roux-en-Y gastric bypass: a review of 70 patients. Obes Surg. 2004;14:1349-53.

38. Weber M, Müller MK, Michel JM, et al. Laparoscopic Roux-en-Y gastric bypass, but not rebanding, should be proposed as rescue procedure for patients with failed laparoscopic gastric banding. Ann Surg. 2003;238:827-34.

39. Shouten R, Van Dielen FMH, Van Gemert WG, et al. Conversion of vertical banded gastroplasty to Roux-en-Y gastric bypass results in restoration of the positive effect on weight loss and co-morbidities: evaluation of 101 patients. Obes Surg. 2007;17:622-30.
40. 12The Longitudinal Assessment of Bariatric Surgery (LABS) Consortium. Perioperative safety in the longitudinal assessment of bariatric surgery. N Engl J Med. 2009;361:445-54.

41. Suter M, Donadini A, Romy S, et al. Laparoscopic Roux-en-Y gastric bypass: significant long-term weight loss, improvement of obesity-related comorbidities and quality of life. Ann Surg. 2011;254:267-73.

42. Skroubis G, Karamanakos S, Sakellaropoulos G, et al. Comparison of early and late complications after various bariatric procedures: incidence and treatment during 15 years at a single institution. World J Surg. 2011;35:93-101.

43. Van Nieuwenhove Y, Dambrauskas Z, Campillo-Soto A, et al. Preoperative very low-calorie diet and operative outcome after laparoscopic gastric bypass. A randomized multicenter study. Arch Surg. 2011;146:1300-5.

44. Vilallonga R, Fort JM, Gonzalez O, et al. Management of patients with hernia or incisional hernia undergoing surgery for morbid obesity. J Obes. 2011; 2011:860942. Epub 2010 Dec 5.

45. Schuster R, Curet MJ, Alami RS, et al. Concurrent gastric bypass and repair of anterior abdominal wall hernias. Obes Surg. 2006; $16: 1205-8$

46. Baltasar A. Mucocele of the gastric tube after conversion of VBG to DS. Obes Surg. 2006;16:528.

47. Sánchez-Pernaute A, Pérez-Aguirre E, Talavera P, et al. Mucocele of the gastric tube after conversion of vertical banded gastroplasty to duodenal switch: not just a radiological image. Obes Surg. 2006;16:524-7. 